\title{
Administração repetida de doses não tóxicas de monofluoroacetato de sódio não protege contra a intoxicação por este composto em ovinos ${ }^{1}$
}

\begin{abstract}
Ariany C. Santos ${ }^{2}$, Franklin Riet-Correa ${ }^{3}$, Rubiane F. Heckler ${ }^{2}$, Stephanie C. Lima ${ }^{4}$, Mariana L. Silva ${ }^{4}$, Renato Rezende ${ }^{5}$, Nilton M. Carvalho ${ }^{6}$ e Ricardo A.A. Lemos ${ }^{6 *}$

ABSTRACT.- Santos A.C., Riet-Correa F., Heckler R.F., Lima S.C., Silva M.L., Rezende R., Carvalho N.M. \& Lemos R.A.A. 2014. [Repeated administration of non-toxic doses of sodium monofluoroacetate does not protect against poisoning by this compound in sheep.] Administração repetida de doses não tóxicas de monofluroacetato de sódio não protege contra a intoxicação por este composto em ovinos. Pesquisa Veterinária Brasileira 34(7):649-654. Faculdade de Medicina Veterinária e Zootecnia, Universidade Federal de Mato Grosso do Sul, Av. Senador Filinto Müller 2443, Campo Grande, MS 79074-460, Brazil. E-mail: lap.famez@ufms.br

With the objective to assess whether repeated non-toxic doses of sodium monofluoroacetate (MFA) induce resistance to poisoning by this compound, 18 sheep were randomly divided into two experimental groups of nine animals each. Sheep from Group 1 ingested non-lethal increasing doses of MFA for six periods: $0.05 \mathrm{mg} / \mathrm{kg}$ for 5 days; $0.08 \mathrm{mg} / \mathrm{kg}$ for 4 days; $0.08 \mathrm{mg} / \mathrm{kg}$ for 4 days; $0.1 \mathrm{mg} / \mathrm{kg}$ for 3 days; $0.1 \mathrm{mg} / \mathrm{kg}$ for 3 days and $0.25 \mathrm{mg} / \mathrm{kg}$ for 3 days. Between the first and second period of administration and between the second and third period the animals did not receive MFA for 10 consecutive days, between the third and fourth period and during the remaining periods of administration the sheep were left 15 days without ingesting MFA. Group 2 was not adapted to the ingestion of MFA and received a single dose of $1 \mathrm{mg} / \mathrm{kg}$ of MFA at the same time that Group 1 was challenged. After challenge, seven sheep of Group 1 showed clinical signs of poisoning and one sheep recovered. In Group 2 , all animals showed clinical signs of poisoning by MFA, however two sheep recovered. The mortality rate was $66.6 \%$ in Group 1 and $77.7 \%$ for Group 2 . These results suggest that repeated administration of non-toxic doses of MFA does not protect against acute poisoning by this compound; therefore other alternatives of prophylaxis for poisoning by plants containing MFA should be searched, mainly the use of intraruminal bacteria that hydrolyze MFA.
\end{abstract}

INDEX TERMS: Sodium Monofluoroacetate, plant poisoning, Palicourea spp., Amorimia spp., Tanaecium bilabiatum, induced resistance, sheep.

RESUMO.- Com o objetivo de avaliar se repetidas doses não tóxicas de monofluoroacetato de sódio (MFA) induzem resistência à intoxicação por essa substância, 18 ovinos foram distribuídos aleatoriamente em dois grupos experimentais de nove animais cada. Os ovinos do Grupo 1 ingeriram doses crescentes não letais de MFA por seis períodos: 0,05mg/

\footnotetext{
${ }^{1}$ Recebido em 21 de maio de 2014.

Aceito para publicação em 16 de junho de 2014.

${ }^{2}$ Programa de Pós-Graduação em Ciência Animal, Faculdade de Medicina Veterinária e Zootecnia (Famez), Universidade Federal de Mato Grosso do Sul (UFMS), Av. Senador Filinto Müller 2443, Campo Grande, MS 79074-460, Brasil.

${ }^{3}$ Laboratório de Anatomia Patológica, Hospital Veterinário, Centro de Saúde e Tecnologia Rural (CSTR), Universidade Federal de Campina Gran-
}

$\mathrm{kg}$ por 5 dias; 0,08mg/kg por 4 dias; 0,08mg/kg por 4 dias; $0,1 \mathrm{mg} / \mathrm{kg}$ por 3 dias; $0,1 \mathrm{mg} / \mathrm{kg}$ por 3 dias e $0,25 \mathrm{mg} / \mathrm{kg}$ por 3 dias. Entre o primeiro e o segundo período de administração e entre o segundo e o terceiro período os animais não receberam o MFA por 10 dias consecutivos; entre o terceiro e o quarto período e dentre os demais períodos de adminis-

\footnotetext{
de (UFCG), Campus de Patos, Avenida Universitária S/N, Santa Cecília, Patos, PB 58700-000, Brasil.

${ }^{4}$ Residência Profissional em Medicina Veterinária, Famez, UFMS, Campo Grande, MS.

${ }^{5}$ Bolsista de Iniciação Científica PIBIC-CNPq, Famez, UFMS, Campo Grande, MS.

${ }^{6}$ Departamento de Medicina Veterinária, Famez/UFMS, Campo Grande, MS. *Autor para correspondência: lap.famez@ufms.br
} 
tração, os ovinos permaneceram 15 dias sem ingerir o MFA. Quinze dias após o último período de administração os ovinos foram desafiados com a dose única de $1 \mathrm{mg} / \mathrm{kg}$ de MFA. 0 Grupo 2 não foi adaptado a ingestão de MFA, estes ovinos receberam dose única de $1 \mathrm{mg} / \mathrm{kg}$ de MFA no mesmo período em que o G1 foi desafiado. No desafio sete ovinos do Grupo 1 apresentaram sinais clínicos da intoxicação e um ovino se recuperou. No Grupo 2 todos os animais manifestaram quadro clínico da intoxicação por MFA, no entanto, dois ovinos se recuperaram. Os coeficientes de mortalidade foram de 66,6\% para o Grupo 1 e de $77,7 \%$ para o Grupo 2. Os resultados deste trabalho sugerem que a administração repetida de doses não tóxicas de MFA não protege contra a intoxicação aguda por este composto, portanto, outras alternativas para a profilaxia da intoxicação por plantas que contêm MFA deverão ser pesquisadas, principalmente a utilização intraruminal de bactérias que hidrolisam MFA.

TERMOS DE INDEXAÇÃO: Monofluoroacetato de sódio, intoxicação por plantas, Palicourea spp., Amorimia spp., Tanaecium bilabiatum, indução de resistência, ovinos.

\section{INTRODUÇÃO}

O monofluoroacetato de sódio (MFA) é uma substância extremamente tóxica para muitas espécies animais (Omara \& Sisodia 1990) e já foi identificado em diversas plantas na África do Sul (Marais 1944, Vickery et al. 1973, Nwude et al.1977) e na Austrália (McEwan 1964, Everist 1974, Baron et al. 1987). A toxicidade do MFA ocorre, exclusivamente, pela ação do fluorocitrato, um metabólito tóxico formado no organismo por meio da denominada "síntese letal". 0 fluorocitrato bloqueia competitivamente a aconitase (Krebs et al. 1994) e impede a conversão do citrato em isocitrato, resultando no acúmulo de grandes quantidades de citrato nos tecidos. Com isso, há diminuição da produção de ATP e os processos metabólicos dependentes de energia são bloqueados (Peters 1952). Descreve-se que em ruminantes a morte sobrevenha pelo efeito intenso sobre o coração (Schultz et al. 1982, Jubb et al. 2007).

No Brasil, MFA foi isolado em Palicourea marcgravii (Oliveira 1963, Cook et al. 2014), Palicourea aeneofusca (Lee et al. 2012, Cook et al. 2014), Palicourea amapaensis, Palicourea grandiflora, Palicourea aff. juruana, Palicourea longiflora, Palicourea aff. longiflora, Palicourea macarthurorum, Palicourea nigricans e Palicourea vacillans (Cook et al. 2014), Amorimia rigida (Cunha et al. 2012, Lee et al. 2012), Amorimia septentrionalis e Amorimia pubiflora (Lee et al. 2012), e Tanaecium bilabiatum (Arrabidaea bilabiata) (Krebs et al. 1994). Plantas conhecidas por causarem insuficiência cardíaca aguda em ruminantes são responsáveis por cerca de 500.000 mortes de bovinos por ano (Tokarnia et al. 2012). Em bovinos, descrevem-se as seguintes doses tóxicas para algumas dessas plantas: $0,5-2,0 \mathrm{~g} / \mathrm{kg}$ para P. marcgravii (Barbosa et al. 2003), $0,75 \mathrm{~g} / \mathrm{kg}$ para $P$. aeneofusca(Tokarnia et al. 1983), 5,0-20,0g/kg para $A$. pubiflora (Tokarnia et al. 1973), 5-10g/kg para A. exotropi$c a$ e $0,65-2,5 \mathrm{~g} / \mathrm{kg}$ para $A$. aff. rigida (Tokarnia et al. 1985). Para ovinos, experimentalmente, relatam-se as doses tóxicas para as plantas subsequentes: P. marcgravii $0,5-1,0 \mathrm{~g} /$ $\mathrm{kg}$ (Tokarnia et al. 1986), A. pubiflora1,5-20g/kg (Becker et al. 2013) e A. septentrionalis $5,0-20 \mathrm{~g} / \mathrm{kg}$ (Vasconcelos et al. 2008).

Na Austrália, a possibilidade de prevenir intoxicações por MFA em ruminantes através da inoculação ruminal de bactérias geneticamente modificadas, contendo um gene que codifica uma fluoroacetato dehalogenase tem sido investigada (Gregg et al. 1994, Gregg et al. 1998). No Brasil, estudos para implantar medidas de controle e profilaxia efetivas para as intoxicações por plantas que contenham MFA encontram-se em desenvolvimento pelo Instituto Nacional de Ciência e Tecnologia para o Controle das Intoxicações por Plantas (INCTCIP). Em trabalhos realizados por este grupo de pesquisa foi demonstrado que a administração diária e contínua de doses não tóxicas de $A$. septentrionalis (Duarte et al. 2013) e P. aeneofusca (Oliveira et al. 2013) administradas em períodos alternados, o que permite a detoxificação do MFA, induz resistência contra intoxicações por essas plantas. Descreve-se, também, que esta resistência pode ser transmitida de um animal resistente para um animal suscetível mediante a transfaunação de conteúdo ruminal (Duarte et al. 2013). Esses resultados somados aos estudos realizados por Camboim et al. (2012a,b) sugerem algumas possibilidades a serem investigadas para o controle das intoxicações por plantas que contenham MFA: 1) administração repetida, por períodos alternados, de doses não tóxicas da planta; 2) administração de MFA em doses não tóxicas que permita a proliferação de bactérias que tenham atividade de dehalogenases; e 3) administração de outro substrato, não tóxico, que estimule a proliferação de bactérias com atividade de dehalogenases. Até o momento, não há estudos que avaliem capacidade de se induzir resistência à intoxicação utilizando o MFA, uma substância de alta toxicidade.

0 presente trabalho tem como objetivo avaliar se é possível induzir resistência à intoxicação por MFA em ovinos mediante a administração de doses crescentes não letais de MFA por períodos alternados, desafiando-os com dose tóxica única do princípio tóxico.

\section{MATERIAL E MÉTODOS}

Para a realização do experimento, foram utilizados 18 ovinos divididos aleatoriamente em dois grupos. Os ovinos eram provenientes de áreas sem a ocorrência da intoxicação por plantas do gênero Amorimia, sem raça definida, sendo 16 machos não castrados e duas fêmeas, com cinco a sete meses de idade e pesos entre 20-40 kg, previamente vermifugados (Nitroxinil 34\%). Os ovinos foram mantidos em baias individuais com área de $2,0 \mathrm{~m}^{2}$, alimentados com ração comercial para a espécie (equivalente a $1 \%$ do peso vivo), feno de Cynodon dactylon (Tifton), sal mineral e água à vontade. Cada ovino recebeu um número de identificação (Ovinos 1-18) e foi submetido ao exame clínico completo antes da administração do MFA.

O Grupo 1 (G1) foi constituído por nove ovinos machos, não castrados, que receberam doses crescentes não letais de MFA por seis períodos: $0,05 \mathrm{mg} / \mathrm{kg}$ por 5 dias; $0,08 \mathrm{mg} / \mathrm{kg}$ por 4 dias; $0,08 \mathrm{mg} / \mathrm{kg}$ por 4 dias; $0,1 \mathrm{mg} / \mathrm{kg}$ por 3 dias; $0,1 \mathrm{mg} / \mathrm{kg}$ por 3 dias e $0,25 \mathrm{mg} / \mathrm{kg}$ por 3 dias. Entre o primeiro e o segundo período de administração e entre o segundo e o terceiro período os animais não receberam o MFA por 10 dias consecutivos; entre o terceiro 
e o quarto período e dentre os demais períodos de administração, os ovinos permaneceram 15 dias sem ingerir o MFA. Durante cada período de fornecimento do MFA foram realizados exames clínicos que consistiam em verificação das frequências cardíaca e respiratória, da temperatura retal, da motilidade ruminal e do comportamento do animal. Quinze dias após o último período de administração os ovinos foram desafiados com a dose única de $1 \mathrm{mg} / \mathrm{kg}$ de MFA. As doses administradas foram estabelecidas com base naquelas descritas por Humphreys (1988) e Peixoto et al. (2010); a metodologia foi adaptada de Duarte et al. (2013) e Oliveira et al. (2013). O Grupo 2 (G2), considerado como grupo controle positivo, foi composto por sete ovinos machos não castrados e duas fêmeas. Estes ovinos receberam dose única de $1 \mathrm{mg} / \mathrm{kg}$ de MFA no mesmo período em que o G1 foi desafiado.

Durante o período de desafio (dose única de $1 \mathrm{mg} / \mathrm{kg}$ de MFA), os ovinos foram observados em intervalos de 2 horas, no entanto, com o início dos sinais clínicos o intervalo de observação foi reduzido para cada 30 minutos. Não houve movimentação dos animais em nenhum momento após a administração da dose tóxica de MFA.

Para o experimento utilizou-se MFA (Sigma-AldrichCo. ${ }^{7}$ ), com grau de pureza $>95 \%$, diluído no momento da administração com $10 \mathrm{~mL}$ de água destilada. Os ovinos que morreram foram necrop- siados imediatamente após a morte, com a coleta de todos os órgãos, fixados em formol tamponado a 10\%. Após a fixação, os fragmentos foram clivados, desidratados em álcool etílico absoluto, diafanizados em xilol, incluídos em parafina e cortados em micrótomo à espessura de $4 \mu$, e os cortes corados pela hematoxilina-eosina (HE) para exame histopatológico. Para o cálculo de mortalidade foram avaliados os grupos G1 e G2 separadamente, considerando a relação entre a frequência absoluta de mortes e o número de animais sob risco.

0 experimento foi aprovado pela Comissão de Ética no Uso de Animais (CEUA) da UFMS e protocolado sob o nํ5 537/2013.

\section{RESULTADOS}

No Grupo 1, durante os cinco primeiros períodos de indução de resistência nenhum animal apresentou sinais clínicos de intoxicação por MFA. No último período $(0,25 \mathrm{mg} / \mathrm{kg}$ de MFA por 3 dias), os Ovinos 1 e 3 apresentaram apatia, hiporexia a anorexia, leves tremores nos membros posteriores, cabeça e pescoço, micção frequente, ataxia, espas-

\footnotetext{
${ }^{7}$ Sigma-Aldrich, 3050 Spruce Street, St Louis, MO 63103, USA <http:// www.sigma-aldrich.com>.
}

Quadro 1. Principais dados sobre o período de desafio, evolução, sinais clínicos e desfecho da indução de resistência à ingestão de monofluoroacetato de sódio (MFA) em ovinos

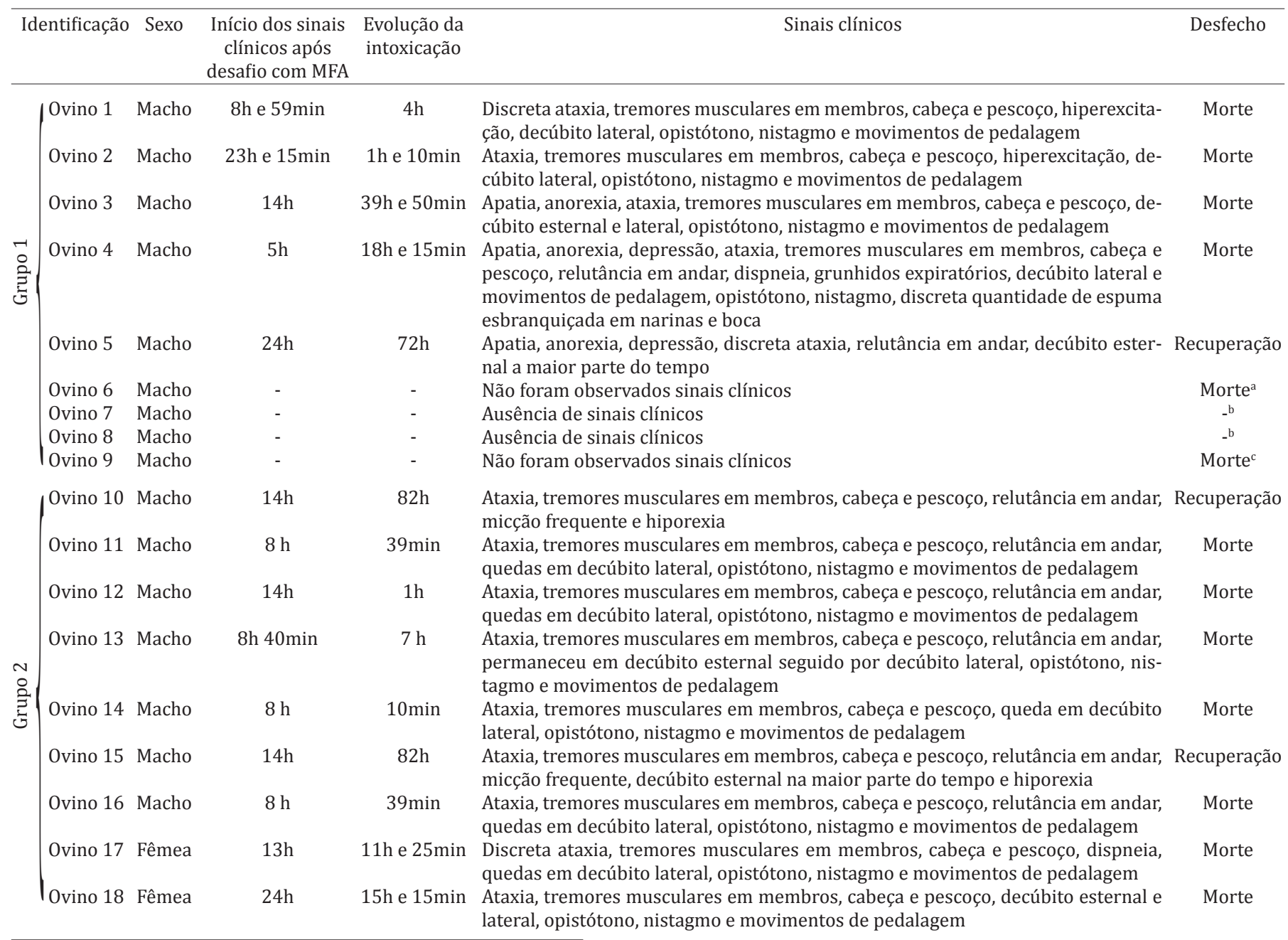

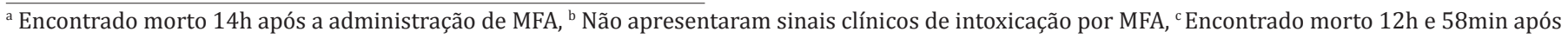
a administração de MFA. 
ticidade, relutância em andar e decúbito esternal a maior parte do tempo. Os sinais clínicos foram observados cerca de 14 horas e 7 horas, respectivamente, após a terceira dose de $0,25 \mathrm{mg} / \mathrm{kg}$ de MFA. Os Ovinos 1 e 3 mostraram melhora do quadro clínico com recuperação 58 horas e 66 horas, respectivamente, após a terceira administração de MFA. Estes animais foram mantidos no experimento para o período de desafio.

No período de desafio com dose única de $1,0 \mathrm{mg} / \mathrm{kg}$ de MFA, para o Grupo 1, o tempo decorrido entre a administração e a manifestação dos primeiros sinais clínicos variou de $5 \mathrm{~h}$ a $24 \mathrm{~h}$. A evolução da intoxicação foi de $1 \mathrm{~h} 10 \mathrm{~min}$ a 39 h50min para os Ovinos 1, 2, 3 e 4. Em dois animais (Ovinos 6 e 9) não foram observados sinais clínicos da intoxicação por MFA, no entanto, foram encontrados mortos $14 \mathrm{~h}$ e 12h58min, respectivamente, após a administração do princípio tóxico. Apenas um animal (Ovino 5) demonstrou recuperação do quadro clínico com cerca de $72 \mathrm{~h}$ após os primeiros sinais da intoxicação. Os Ovinos 7 e 8 não manifestaram sinais clínicos da intoxicação por MFA, conforme o Quadro 1.

Nos sete ovinos do Grupo 1 em que foram observados sinais clínicos, verificaram-se apatia, anorexia, ataxia, tremores musculares em membros, cabeça e pescoço, depressão, relutância em andar e dificuldade quando iam deitar-se, dispneia e grunhidos expiratórios, hiperexcitação e decúbito esternal a maior parte do tempo. Na fase final, em geral, os animais caíam em decúbito lateral com movimentos de pedalagem, apresentavam respiração ofegante, opistótono, nistagmo, esticavam os membros e morriam (Quadro 1).

No Grupo 2, o intervalo entre a administração do MFA e a manifestação dos sinais clínicos ocorreu entre 8h e 24h, com evolução clínica de $39 \mathrm{~min}$ a $15 \mathrm{~h} 15 \mathrm{~min}$. Dois animais (Ovinos 10 e 15) apresentaram recuperação do quadro clínico 82h após demonstrarem sinais de intoxicação. Os sinais clínicos consistiam em ataxia, tremores musculares em membros, cabeça e pescoço, micção frequente, hiporexia, relutância em andar, decúbito esternal a maior parte do tempo, quedas em decúbito lateral com movimentos de pedalagem, opistótono e nistagmo (Quadro 1).

As alterações macroscópicas foram semelhantes para o Grupo 1 e o Grupo 2, caracterizadas por aurículas, veias cavas caudal e cranial, jugulares, ázigos, costo cervical, ilíacas e pulmonares leve a acentuadamente ingurgitadas, discreto a moderado hidropericárdio e raras petéquias no pericárdio. Os pulmões apresentavam-se não colapsados ("armados"), lisos, brilhantes, pesados, com áreas irregulares avermelhadas e discretamente congestos. Havia discreta quantidade de espuma esbranquiçada na traquéia próximo a bifurcação dos brônquios. 0 fígado estava acastanhado intercalado com áreas irregulares avermelhadas. Os rins apresentavam cortical pálida ao corte em sete animais (Ovinos $1,4,9,11,12,13$ e 16) e nos demais, cortical e medular difusamente avermelhadas. No miocárdio do Ovino 3 havia áreas pálidas.

Os principais achados histopatológicos foram observados no rim, fígado e coração conforme Quadro 2. No rim, de todos os ovinos avaliados, havia discreta a moderada deposição de material amorfo eosinofílico na luz de túbulos contorcidos e no espaço capsular. Apenas oito ovinos (Ovinos 2, 3, 4, 6, 12, 13, 17 e 18) apresentaram discreta a moderada tumefação e vacuolização (degeneração hidrópico-vacuolar) dos túbulos contorcido distais associadas à picnose nuclear. Havia ainda, discreta a moderada tumefação e vacuolização dos túbulos contorcidos proximais (Ovinos 1, 2, 3, 9, 17 e 18), múltiplos focos discretos de infiltrado linfoplasmocitário intersticial (Ovinos 1 e 16) e discreta a moderada congestão. No fígado, em geral, observaram-se discreta a acentuada tumefação e vacuolização difusa dos hepatócitos e discreta necrose individual aleatória de hepatócitos. Em um animal (Ovino 18) havia cirrose hepática associada a cristais birrefringentes na luz de ductos biliares e macrófagos espumosos, no entanto, este ovino não apresentava sinais clínicos de intoxicação por Brachiaria spp. No coração de nove ovinos (Ovinos 1, 3, 4, 6, 9, 12, 13, 14 e 16) havia discreta vacuolização intracitoplasmática

Quadro 2. Principais alterações histopatológicas e intensidade das lesões observadas nos rins, fígado e coração dos ovinos intoxicados por monofluoroacetato de sódio (MFA) durante o período desafio, da indução de resistência à ingestão de MFA em ovinos

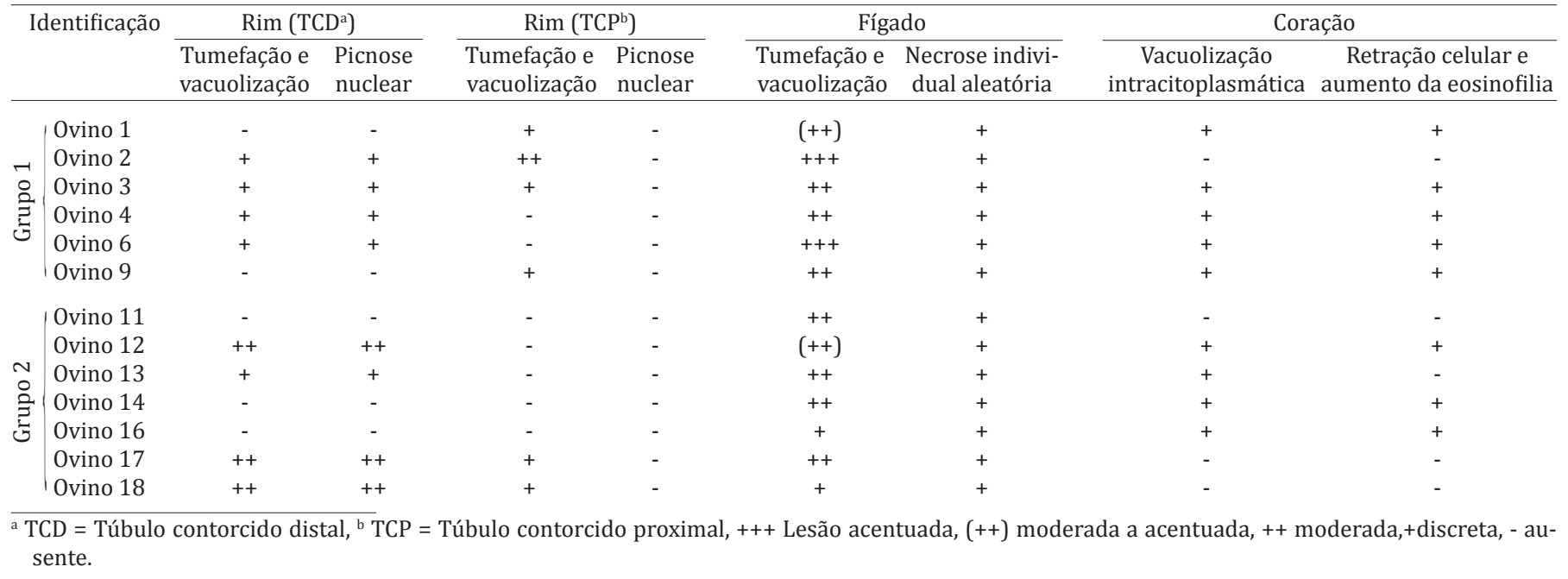


das fibras musculares e discreta retração celular, aumento da eosinofilia e picnose nuclear em fibras musculares individuais aleatórias. No cerebelo e em fragmentos de córtex do Ovino 18, observaram-se discretas formações vacuolares de diferentes tamanhos na substância branca próximo a junção com a substância cinzenta. Nos demais órgãos não foram encontradas alterações significativas.

Os coeficientes de mortalidade foram de $66,6 \%$ para o Grupo 1 e de $77,7 \%$ para o Grupo 2.

\section{DISCUSSÃO E CONCLUSÃO}

A metodologia utilizada não foi eficiente para induzir a resistência à intoxicação por MFA em ovinos. Ainda que, no Grupo 1 três ovinos tenham sobrevivido, dos quais, os Ovinos 7 e 8 não apresentaram nenhuma manifestação clínica de intoxicação e o Ovino 5 recuperou-se em 72 horas após os primeiros sinais da intoxicação, o coeficiente de mortalidade foi de $66,6 \%$, enquanto para o Grupo2 esse coeficiente foi de 77,7\%. Em estudos anteriores para indução de resistência a intoxicação por A. septentrionalis (Duarte et al. 2013) e P. aeneofusca (Oliveira et al. 2013), com a administração de doses não letais crescentes dessas plantas, nenhum caprino morreu durante ou após o período de desafio. Em ambos os experimentos, todos os animais do grupo controle adoeceram e houve $33,3 \%$ de mortalidade nesse grupo controle, no qual os caprinos não tinham sido adaptados ao consumo da planta.

Embora neste estudo a presença de bactérias que degradem o MFA não tenha sido avaliada, este é o principal mecanismo proposto para a ocorrência da resistência a intoxicação por plantas que tem o MFA como princípio tóxico (Duarte et al. 2013). Os resultados obtidos no presente estudo demonstram que existem ovinos naturalmente resistentes a intoxicação corroborando com as observações de relatos anteriores em que animais criados em áreas livres da planta podem conter bactérias que degradam o MFA em seu rúmen (Camboim et al. 2012b). Porém, a administração de doses não letais crescentes de MFA não foram capazes de provocar o crescimento da população destas bactérias ao ponto de induzir a resistência ao desafio com 1,0 mg/Kg de MFA. Estudos realizados na Austrália demonstram que a inoculação no rúmen de uma estirpe geneticamente modificada de Butyrivibrio fibrisolvens com um gene que codifica uma fluoroacetato dehalogenase, proveniente de uma espécie de Moraxella, foi eficiente em prevenir a intoxicação por MFA em ovinos (Gregg et al. 1998).

A dose tóxica de MFA empregada durante o período de desafio corresponderia a $16,6 \mathrm{~g} / \mathrm{kg}$ de $A$. pubiflora, se consideramos a concentração de $0,006 \%$ de MFA encontrada em amostras de A. pubiflora mantidas no Herbário da Universidade de Michigan (Lee et al. 2012). Desta forma, a dose tóxica utilizada estaria de acordo com a variação de toxidez da planta observada para a espécie bovina $(0,5-20,0 \mathrm{~g} / \mathrm{kg})$ e ovina $(1,5-20,0 \mathrm{~g} / \mathrm{kg})$ (Tokarnia et al. 1973, Becker et al. 2013). Ressalta-se que no estado de Mato Grosso do Sul a A. pubiflo$r a$ é uma das plantas tóxicas mais importantes, responsável por surtos de morte súbita em bovinos (Lemos et al. 2011).

Graus variáveis de degeneração hidrópico-vacuolar ocorreram em $61,5 \%$ dos ovinos deste estudo, diferente do observado por Peixoto et al. (2010) em que todos os ovinos intoxicados com MFA apresentaram lesão renal. Esta não é uma alteração específica, mas pode ser um achado diagnóstico em animais intoxicados por plantas que contêm esse princípio tóxico (Oliveira et al. 2004, Helayel et al. 2009, Nogueira et al. 2010, Tokarnia et al. 2012). As lesões hepáticas de tumefação e vacuolização foram observadas em todos os ovinos, com intensidade discreta a moderada e distribuição difusa. Alterações semelhantes foram descritas em um ovino intoxicado por MFA, no entanto, a lesão se restringia, predominantemente, a regiões periportais (Peixoto et al. 2010). No coração de oito ovinos havia discreta necrose de coagulação de fibras musculares individuais, caracterizada por retração celular, aumento da eosinofilia do citoplasma com perda das estriações e núcleos picnóticos, além de vacúolos intracitoplasmáticos observados em algumas células. Esta alteração já foi descrita em bovinos (Pavarini et al. 2012) e caprinos (Oliveira et al. 2013) intoxicados por plantas que contêm o MFA. Em bovinos intoxicados por A. exotropica descreve-se também áreas multifocais e coalescentes de fibrose cardíaca (Soares et al. 2012).

Em trabalhos anteriores foi comprovado que a administração de doses não tóxicas de plantas que contem MFA aumentam a resistência contra a intoxicação por essas plantas e foi elaborada a hipótese de que doses não toxicas de MFA poderiam também aumentar a resistência a intoxicação por essa substância (Duarte et al. 2013, Oliveira et al. 2013). Os resultados deste trabalho sugerem que a administração repetida de doses não tóxicas de MFA não protege contra a intoxicação aguda por esta substância, portanto, outras alternativas para o controle das intoxicações por plantas que contêm MFA deverão ser pesquisadas, principalmente a utilização de bactérias que hidrolisam MFA.

Agradecimentos.- À Fundação de Apoio ao Desenvolvimento do Ensino, Ciência e Tecnologia do Estado de Mato Grosso do Sul (Fundect/CAPES no 011/2013, Doutorado em MS) pela concessão de bolsa; ao Conselho Nacional de Desenvolvimento Científico e Tecnológico (CNPq no 14/2013, Projeto Universal Proc. 480550/2013-1) e ao Instituto Nacional de Ciência e Tecnologia para o Controle das Intoxicações por Plantas (INCTCIP) pelo apoio financeiro.

\section{REFERÊNCIAS}

Barbosa J.D., Oliveira C.M.C., Tokarnia C.H. \& Riet-Correa F. 2003. Comparação da sensibilidade de bovinos e búfalos à intoxicação por Palicourea marcgravii (Rubiaceae). Pesq. Vet. Bras. 23(4):167-172.

Baron M.L., Bothroyd C.M., Rogers G.I., Staffa A. \& Rae I.D. 1987. Detection and measurement of fluoroacetate in plant extracts by F-NMR. Phytochem. 26(8):2293-2295.

Becker M., Caldeira F.H.B., Carneiro F.M., Oliveira L.P., Tokarnia C.H., RietCorrea F., Lee S.T. \& Colodel E.M. 2013. Importância da intoxicação por Amorimia pubiflora (Malpighiaceae) em bovinos em Mato Grosso: reprodução experimental da intoxicação em ovinos e bovinos. Pesq. Vet. Bras. 33(9):1049-1056.

Camboim E.K.A., Tadra-Sfeir M.Z., Souza E.M., Pedrosa F.O., Andrade P. P., McSweeney C.S., Riet-Correa F. \& Melo M.A. 2012a. Defluorination of sodium fluoroacetate by bacteria from soil and plants in Brazil. Scientific World Journal 2012:149893.

Camboim E.K.A., Almeida A.P., Tadra-Sfeir M.Z., Junior F.G., Andrade P.P., McSweeney C.S., Riet-Correa F. \& Melo M.A. 2012b. Isolation of sodium fluoroacetate degrading bacteria from caprine rumen in Brazil. Scientific World Journal 2012:178254 
Cook D., Lee S.T., Taylor C.M., Bassüner B., Riet-Correa F., Pfister J.A. \& Gardner D.R. 2014. Detection of toxic monofluoroacetate in Palicourea species. Toxicon 80:9-16.

Cunha L.C., Pipole F., Carvalho L.R., Lago J.H.G. \& Görniak S.L. 2012. Isolation and characterization of sodium 2-fluoroacetate from Mascagnia rigida using chromatography andinfrared spectroscopy. Toxicon 60:329-332.

Duarte A.L.L., Medeiros R.M.T., Carvalho F.K.L., Lee S.T., Cook D., Pfister J.A., Costa V.M.M. \& Riet-Correa F. 2013. Induction and transfer of resistance to poisoning by Amorimia (Mascagnia) septentrionalis in goats J. Appl. Toxicol. Doi 10.1002/jat.2860

Everist S.L. 1974. Poisonous Plants of Australia. Angus and Robertson Publishers, Sidney. 684p.

Gregg K., Cooper C.L., Schafer D.J., Sharpe H., Beard C.E., Allen G. \& Xu J. 1994. Detoxification of the plant toxin fluoroacetate by a genetically modified rumen bacterium. Nature 12(13):1361-1365.

Gregg K., Hamdorf B., Henderson K., Kopecny J. \& Wong C. 1998. Genetically modified ruminal bacteria protect sheep from fluoroacetate poisoning. Appl. Environ. Microbiol. 64(9):3496-3498.

Helayel M.A., França T.N., Seixas J.N., Nogueira V.A., Caldas S.A. \& Peixoto P.V. 2009. Morte súbita em bovinos causada pela ingestão de Pseudocalymma elegans (Bignoniaceae) no município de Rio Bonito, RJ. Pesq. Vet. Bras. 29(7):498-508.

Humphreys D.J. 1988. Veterinary Toxicology. $3^{\text {rd }}$ ed. Bailliere Tindall, London. 356p.

Jubb K.V.F., Kennedy P.C. \& Palmer N. 2007. Pathology of Domestic Animals. Vol.3, $5^{\text {th }}$ ed. Saunders Elsevier, Toronto. 737p.

Krebs H.C., Kemmerling W. \& Habermehl G. 1994. Qualitative and quantitative determination of fluoroacetic acid in Arrabidaea bilabiata and Palicourea marcgravii by F-19-NMR Spectroscopy. Toxicon 32(6):909-913.

Lee S.T., Cook D., Riet-Correa F., Pfister J.A., Anderson W.R., Lima F.G. \& Gardner D. 2012. Detection of monofluoracetate in Palicourea and Amorimia species. Toxicon 60:791-796.

Lemos R.A.A., Guimarães E.B., Carvalho N.M., Nogueira A.P.A., Santos B.S., Souza R.I.C., Cardinal S.G. \& Kassab H.O. 2011. Plant poisonings in Mato Grosso do Sul, p.68-72. In: Riet-Correa F., Pfister J., Schild A.L. \& Wierenga T. (Eds), Poisoning by Plants, Mycotoxins, and Related Toxins. CAB International, Wallingford, UK.

Marais J.S.C. 1944. Monofluoroacetic acid, the toxic principle of "gifblaar" Dichapetalum cymosum (Hokk) Engl. Onderstepoort J. Vet. Sci. Anim. Ind. 20(1):67-73.

McEwan T. 1964. Isolation and identification of the principle of Gastrolobium grandiflorum. Queensl. J. Agric. Anim. Sci. 21(2):1-14.

Nogueira V.A., França T.N., Peixoto T.C., Caldas S.A., Armién A.G. \& Peixoto P.V. 2010. Intoxicação experimental por monofluoroacetato de sódio em bovinos: aspectos clínicos e patológicos. Pesq. Vet. Bras. 30(7):533-540.

Nwude N., Parsons L.E. \& Adaudi A.O. 1977. Acute toxicity of the leaves and extracts of Dichapelatum barteri (Engl.) in mice, rabbits and goats. Toxicology 7:23-29.
Oliveira M.M. 1963. Chromatographic isolation of monofluoroacetic acid from Palicourea marcgravii St. Hil. Experientia 19(11):586-587.

Oliveira C.M.C., Barbosa J.D., Macedo R.S.C., Brito M.F., Peixoto P.V. \& Tokarnia C.H. 2004. Estudo comparativo da toxidez de Palicourea juruana (Rubiaceae) para búfalos e bovinos. Pesq. Vet. Bras. 24(1):27-30.

Oliveira M.D., Riet-Correa F., Carvalho F.K.L., Silva G.B., Pereira W.S. \& Medeiros R.M.T. 2013. Indução de resistência à intoxicação por Palicourea aeneofusca (Rubiaceae) mediante administração de doses sucessivas não tóxicas. Pesq. Vet. Bras. 33(6):731-734.

Omara F. \& Sisodia C.S. 1990. Evaluation of potential antidotes for sodium fluoroacetate in mice. Vet. Hum. Toxicol. 32(5):427-431.

Pavarini S.P., Bandinelli M.B., Juffo G.D., Souza S.O., Driemeier D. \& Cruz C.E.F. 2012. Decreased expression of cardiac troponin C is associated with cardiac lesions in Amorimia exotropica poisoned cattle. Pesq. Vet. Bras. 32(10):1005-1008

Peixoto T.C., Nogueira V.A., Coelho C.D., Veiga C.C.P., Peixoto P.V. \& Brito M.F. 2010. Avaliações clínico-patológicas e laboratoriais da intoxicação experimental por monofluoroacetato de sódio em ovinos. Pesq. Vet. Bras. 30(12):1021-1030.

Peters R.A. 1952. Lethal synthesis. Proceedings of the Royal Society of London. Series B, Biological Sciences 139(895):143-170.

Schultz R.A., Coetzer J.A., Kellerman T.S. \& Naudé T.W. 1982. Observations on the clinical, cardiac and histopathological effects of fluoracetate in sheep. Onderstepoort J. Vet. Res. 49(4):237-245.

Soares M.P., Pavarini S.P., Adrien M.L., Quevedo P.S., Schild A.L., Peixoto P.V., Cruz C.E.F. \& Driemeier D. 2011. Amorimia exotropica poisoning as a presumptive cause of myocardial fibrosis in cattle.J. Vet. Diagn. Invest. 23(6):1223-1229.

Tokarnia C.H. \& Döbereiner J. 1973. Intoxicação por Mascagnia pubiflora em bovinos no Estado do Mato Grosso. Pesq. Agropec. Bras. 8:61-68.

Tokarnia C.H., Döbereiner J., Couceiro J.E.M. \& Silva A.C.C. 1983. Intoxicação por Palicourea aeneofusca (Rubiaceae), a causa de "mortes súbitas" em bovinos na Zona-da-Mata de Pernambuco. Pesq. Vet. Bras. 3(3):7579.

Tokarnia C.H., Peixoto P.V. \& Döbereiner J. 1985. Intoxicação experimental por Mascagnia aff. rigida (Malpighiaceae) em bovinos no Norte do Espírito Santo. Pesq. Vet. Bras. 5(3):77-91.

Tokarnia C.H., Peixoto P.V. \& Döbereiner J. 1986. Intoxicação experimental por Palicourea marcgravii (Rubiaceae) em ovinos. Pesq. Vet. Bras. 6(4):121-131.

Tokarnia C.H., Brito M.F., Barbosa J.D., Peixoto P.V. \& Döbereiner J. 2012. Plantas Tóxicas do Brasil. $2^{a}$ ed. Editora Helianthus, Rio de Janeiro. 586p.

Vasconcelos J.S., Riet-Correa F., Dantas A.F.M., Medeiros R.M.T., Galiza G.J.N., Oliveira D.M. \& Pessoa A.F.A. 2008. Intoxicação por Mascagnia rigida (Malpighiaceae) em ovinos e caprinos. Pesq. Vet. Bras. 28(10):521526.

Vickery B., Vickery M.L. \& Ashu J.T. 1973. Analysis of plants for fluoroaceticacidis. Phytochem. 1:145-147. 